\title{
Aircraft Integration and Flight Testing of 4STAR
}

\section{Final Report}
CJ Flynn
P Russell
E Kassianov
S Dunagan
J Redemann
B Holben

October 2012 


\section{DISCLAIMER}

This report was prepared as an account of work sponsored by the U.S. Government. Neither the United States nor any agency thereof, nor any of their employees, makes any warranty, express or implied, or assumes any legal liability or responsibility for the accuracy, completeness, or usefulness of any information, apparatus, product, or process disclosed, or represents that its use would not infringe privately owned rights. Reference herein to any specific commercial product, process, or service by trade name, trademark, manufacturer, or otherwise, does not necessarily constitute or imply its endorsement, recommendation, or favoring by the U.S. Government or any agency thereof. The views and opinions of authors expressed herein do not necessarily state or reflect those of the U.S. Government or any agency thereof. 


\title{
Aircraft Integration and Flight Testing of 4STAR
}

\section{Final Report}

\author{
CJ Flynn E Kassianov \\ P Russell J Redemann \\ St Dunagan B Holben
}

October 2012

Work supported by the U.S. Department of Energy, Office of Science, Office of Biological and Environmental Research 


\section{Summary}

Under funding from the U.S. Dept. of Energy, in conjunction with a funded NASA 2008 ROSES proposal, with internal support from Battelle Pacific Northwest Division (PNWD), and in collaboration with NASA Ames Research Center, we successfully integrated the Spectrometer for Sky-Scanning, SunTracking Atmospheric Research (4STAR-Air) instrument for flight operation aboard Battelle's G-1 aircraft and conducted a series of airborne and ground-based intensive measurement campaigns (hereafter referred to as "intensives") for the purpose of maturing the initial 4STAR-Ground prototype to a flight-ready science-ready configuration.

Following mechanical modifications to the G-1 hatch assembly to accommodate the 4STAR-Air under support from Battelle PNWD, we conducted four intensive campaigns.

Intensive \#1: September 2010. Successful sun-tracking tests aboard the G-1 out of Moffett Field, CA. First atmospheric vertical profile from 4STAR-Air. Identified need for dry air or N2 purge.

Intensive \#2: April 2011. Sun-tracking and sky-scanning airborne tests aboard the G-1 out of Pasco, WA airport. First-ever airborne principal plane and almucanctar sky scans from 4STAR-Air. Successful incorporation of in-flight attitude telemetry and solar ephemeris for tracking recovery in broken clouds, greatly expanding the range of atmospheric conditions conducive to successful operation. Identified need for increased signal levels and improved limit switch reliability.

Intensive \#3: July 2011. Conducted “Sun photometer Inter-Comparison Experiment II" with groundbased tests at NASA Ames, Moffett Field. Demonstrated improved signal levels and developed techniques for airborne characterization of 4STAR-Air.

Intensive \#4: August 2011. Successful execution of all desired airborne operational modes including airborne Langley calibration, sun-tracking and sky-scanning within the boundary layer, above the boundary layer, and between elevated aerosol layers, and both passive and active zenith mode for cloud studies.

Sensitivity Study: In addition, operational AERONET retrieval code was obtained and adapted to facilitate customized retrievals and to conduct a study quantifying the sensitivity of the aerosol intensive property retrievals to errors in measurement or calibration.

Technical Readiness Level Advancement: The 4STAR-Air advanced from a Technical Readiness Level (TRL) of 4 to a final TRL of about 8. Perhaps the most telling indication of the success of this maturation program is the identification of the 4STAR-Air as a critical instrument for participation in the DOE Atmospheric Radiation Measurement (ARM) Climate Research Facility's Two-Column Aerosol Project (TCAP) planned for July 2012 and February 2013. 


\section{Contents}

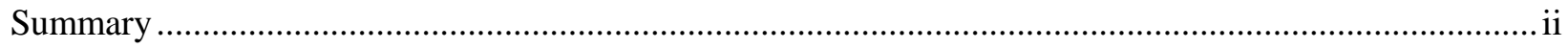

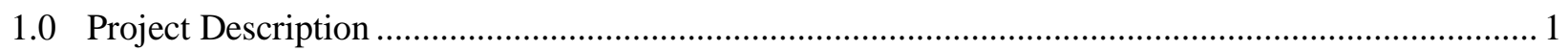

2.0 Selected Results Under Project Funding .................................................................................. 2

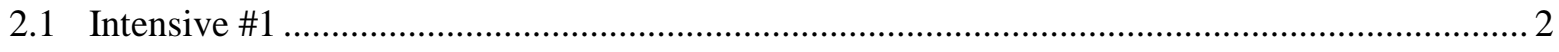

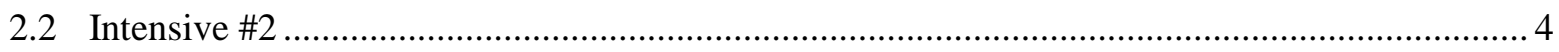

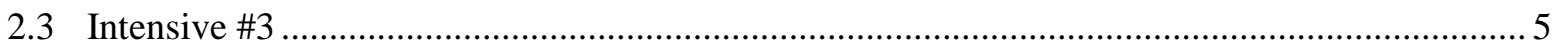

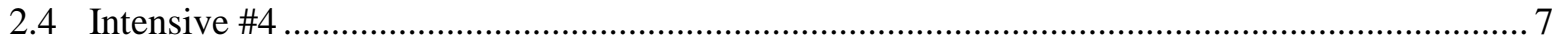

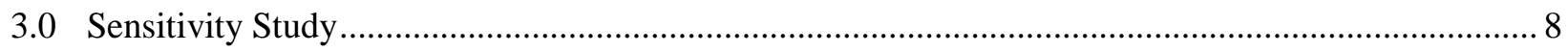

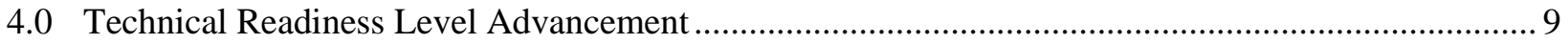

\section{Figures}

1. Flight track of G-1 4STAR test flight on September 28, 2010, out of Moffett Field,

California. 3

2. Tracking data from 4 STAR during flight on September 28, 2010................................................. 4

3. Vertical "optical depth" from airborne profile............................................................................ 4

4. Optical transmission tests of bulk head feed-through......................................................................... 5

5. Assessment of 4STAR sky radiance relative to Prede sunphotometer............................................. 6

6. Angular and spectral uniformity of 4STAR direct sun optics. ..................................................... 7

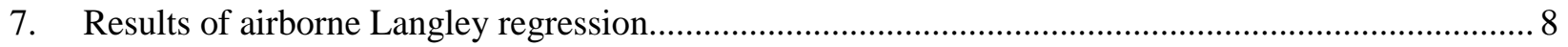

8. Retrieval sensitivity to sky radiance measurement errors............................................................ 8 


\subsection{Project Description}

The goal of the research effort was to develop and demonstrate an airborne Sun-sky spectroradiometer to provide information on aerosols, clouds, and trace gases extending beyond what can be derived from existing airborne sun photometers such as the 14-Channel NASA Ames Airborne Tracking Sunphotometer (AATS-14) and to improve compactness, modularity, and versatility. The enhanced instrument we used was an airborne Spectrometer for Sky-Scanning, Sun-Tracking Atmospheric Research (4STAR-Air) based on the ground prototype (4STAR-Ground) that we have developed and extensively tested over the past several years.

This proposal was supplemental to a companion NASA ROSES 2008 proposal that was selected for funding. The 4STAR instrument concept combines the sun-tracking ability of the current AATS-14 with the sky-scanning ability of the ground-based AERONET sun/sky photometers, while extending both AATS-14 and AERONET measurements by providing full spectral information in 1536 wavelength channels from the UV $(210 \mathrm{~nm})$ to the SWIR $(1690 \mathrm{~nm})$. The full spectral information was used to improve determination of gas phase atmospheric components that in turn can yield improved aerosol measurements. Sky-scanning measurements provided data that can enable retrievals of size distribution modes and also provide information on aerosol type via retrievals of complex refractive index and shape. Cloud property retrievals combining airborne measurements of zenith radiance by 4STAR and upwelling flux by an auxiliary radiometer are better constrained than analogous ground-based techniques. Fast temporal sampling permits clear/cloudy transition studies.

Our proposed approach to providing these enhanced capabilities was to link via fiber optics the suntracking and sky-scanning optical collectors mounted on the exterior of the aircraft to rack-mounted spectrometers housed within the aircraft cabin. This compact modular design yields easier integration on a wider range of aircraft while also affording the potential to substitute or add more specialized spectrometers in future versions.

To demonstrate the feasibility of this approach, we developed and extensively tested a ground-based prototype surpassing critical performance requirements of pointing accuracy, optical throughput and sensitivity, repeatability of fiber optic rotational couplings, and stray light rejection. These tests and our airborne experience with AATS demonstrated that our approach can overcome the challenges of an airborne version (4STAR-Air). To limit costs and increase chances of success, the companion NASA ROSES proposal focused on the limited set of goals comprising the development and demonstration of the airborne prototype 4STAR-Air, but did not include support for final hardening and flight testing of the 4STAR. Therefore, we proposed to integrate the 4STAR into the ARM Aerial Facility (AAF) G-1 under support from ARM. This supplemental investment by ARM highly leveraged the funding provided by both agencies and significantly improved the probability of success. The integration effort included mechanical accommodation as well as electrical and data communication details. In essence, all of the technological challenges we have successfully surpassed with the ground-based instrument were implemented and validated with the airborne system. We conducted flight tests during clear and broken skies to validate the capabilities of the instrument to track the sun under a variety of conditions and to perform programmed sky scans of diffuse sky radiance. Test flight patterns included sequences designed to characterize instrument performance as well as patterns anticipated for science flights including 
ascending and descending spirals as well as level legs at various altitudes within and above the boundary layer. The 4STAR collected direct solar and sky-scanning measurements suitable for retrieving aerosol properties with customized versions of AERONET retrieval code.

\subsection{Selected Results Under Project Funding}

\subsection{Intensive \#1}

On September 28, 2010, we conducted successful sun-tracking tests aboard the G-1 flying out of Moffett Field, CA. A variety of aircraft maneuvers including banking turns, spiral ascents, straight-and-level racetracks, and a slant descent were exercised as shown in the yellow flight track in Figure 1 below. Aircraft telemetry and 4STAR tracking metrics are shown in Figure 2. This flight series yielded the first atmospheric vertical profile from 4STAR-Air, including retrieval of the PBL (planetary boundary layer) total optical depth and optical depth profiles as shown in Figure 3. In conjunction with these tests and through examination of the flight data, we identified a need for dry air or N2 purge of the 4STAR optical head. 


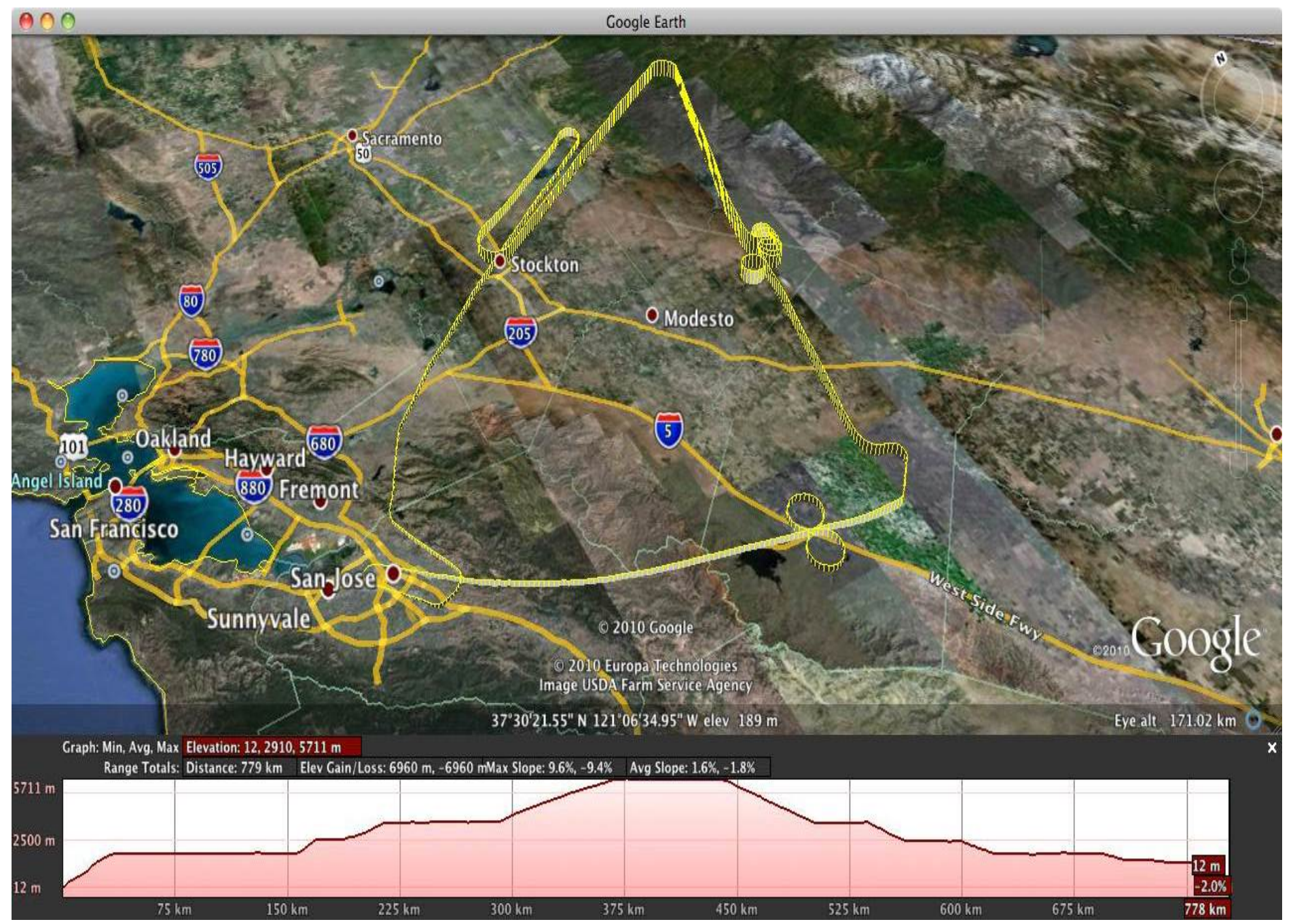

Figure 1. Flight track of G-1 4STAR test flight on September 28, 2010, out of Moffett Field, California. The yellow curtain plot shows the various flight maneuvers executed during this first test flight. The bottom strip chart indicates the flight altitude profile from take-off to landing. 

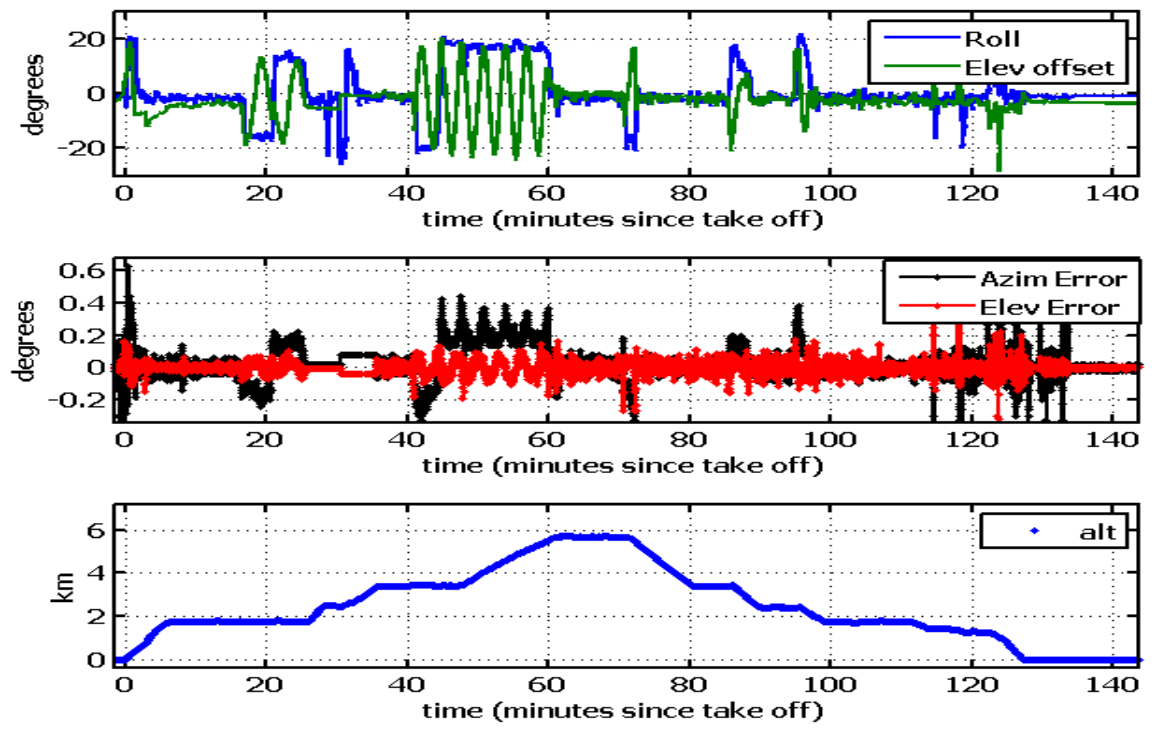

Figure 2. Tracking data from 4STAR during flight on September 28, 2010. The top panel shows the reported aircraft roll (from aircraft attitude telemetry) in blue and the corrective response of the 4STAR elevation motor to track the sun in green. The center panel shows the tracking error derived from quadrant detector signals indicating acceptable tracking $(<0.1$ degree error) when aircraft is not engaged in exceptional maneuvers. The bottom panel shows the altitude profile from take-off to landing.
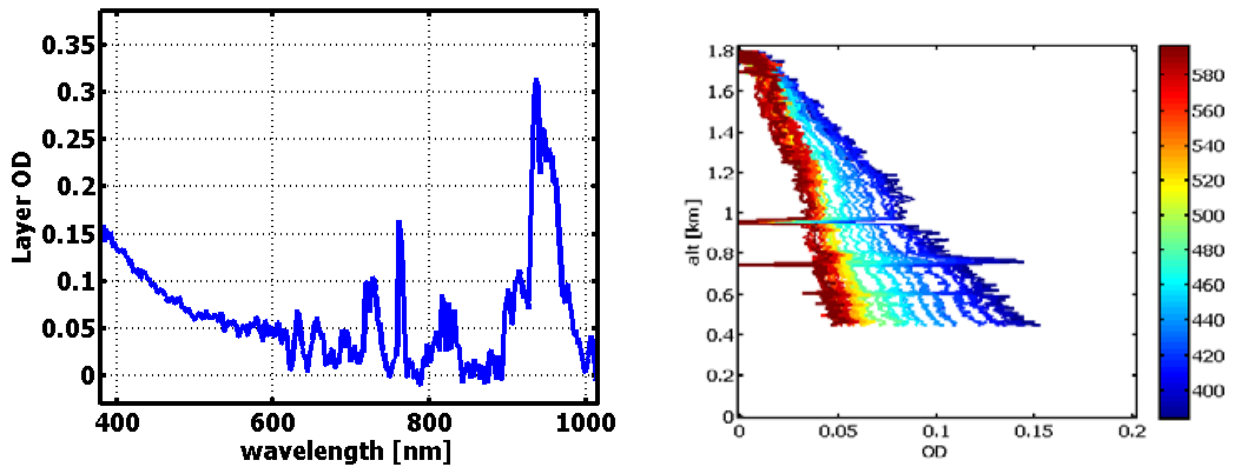

Figure 3. Vertical "optical depth” from airborne profile. Figure 3a on the left shows the log of the total attenuation of the direct sun as function of wavelength from 400 to $1000 \mathrm{~nm}$ in the layer from about 0.5 to $1.8 \mathrm{~km}$ above ground level. Figure $3 \mathrm{~b}$ on the right shows layer optical depth profiles over the same vertical layer but for a selection of spectrometer wavelengths from 380-600 nm.

\subsection{Intensive \#2}

On April 14, 2011, we conducted our second series of test flights, this time out of the Pasco, WA, airport. All desired 4STAR operating modes were tested, including sun-tracking and sky-scanning measurements at a variety of altitudes. Some difficulties associated with limit switch operation at high altitude/cold temperatures were identified. Moreover, although the 4STAR demonstrated the mechanical ability to 
execute all desired modes, post-flight analysis identified the need for increased signal levels. A thorough component-by-component inventory of signal losses (Figure 4) was conducted with observed throughput compared against expectations to identify problem areas and focus effort. Both the limit switch operation and the signal level deficiencies have since been positively remedied.

Additionally, during the April test flight series, we, in coordination with the G-1 flight crew, successfully incorporated real-time telemetry of aircraft attitude and in-flight solar ephemeris calculations to permit near-immediate recovery of the sun even while operating under broken skies. This greatly expands the range of atmospheric conditions through which the 4STAR can operate relative to its predecessor, the AATS-14, and has encouraging implications for the ability of 4STAR to operate productively in a wide range of challenging sky conditions including clear sky, overcast, and broken skies.
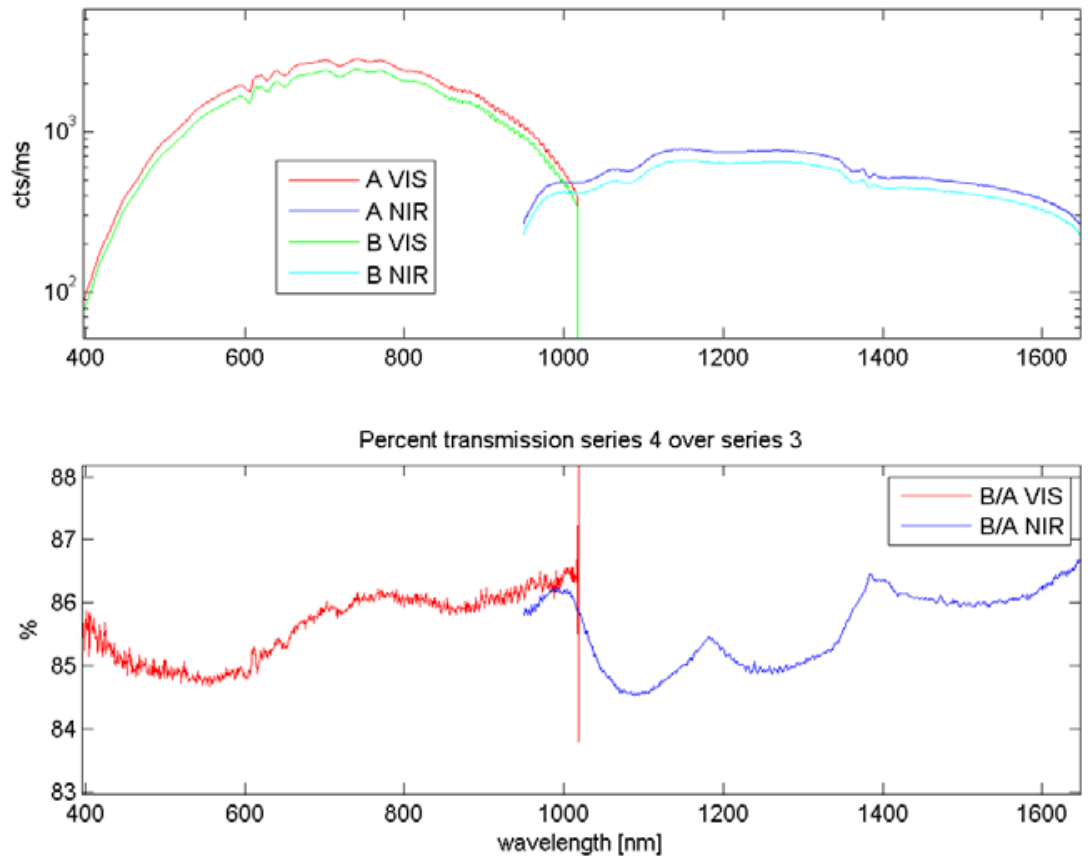

Figure 4. Optical transmission tests of bulk head feed-through. Top panel: the red and blue lines show signal measured as a function of wavelength before the bulkhead feed-through, while the green and cyan lines show the signal measured after the component. The bottom panel shows the ratio between the before and after signal levels in percent with the red line representing the measurements with the CCD spectrometer from 400-1000 nm and the blue line representing the measurements from the InGaAs spectrometer from 970-1650 nm.

\subsection{Intensive \#3}

In mid-July 2011 the 4STAR team conducted extensive ground-based comparisons including the 4STARAir, AATS-14, and a Prede Sun Photometer, and an assortment of lab tests. These tests confirmed the improvement in signal levels from engineering solutions motivated by the previous flight series.

Additionally, the collocated operation permitted radiometric errors in the sky radiance measurements to be quantified as illustrated in Figure 5 and subsequent definition of the sky collector design for the final flight configuration. The intensive period was also used to develop techniques to assess FOV (field-ofview) sensitivity over the entire spectral range of the 4STAR spectrometers (see Figure 6), which led to 
improvements in the spatial and spectral uniformity of the sun collector design implemented in the final flight configuration.

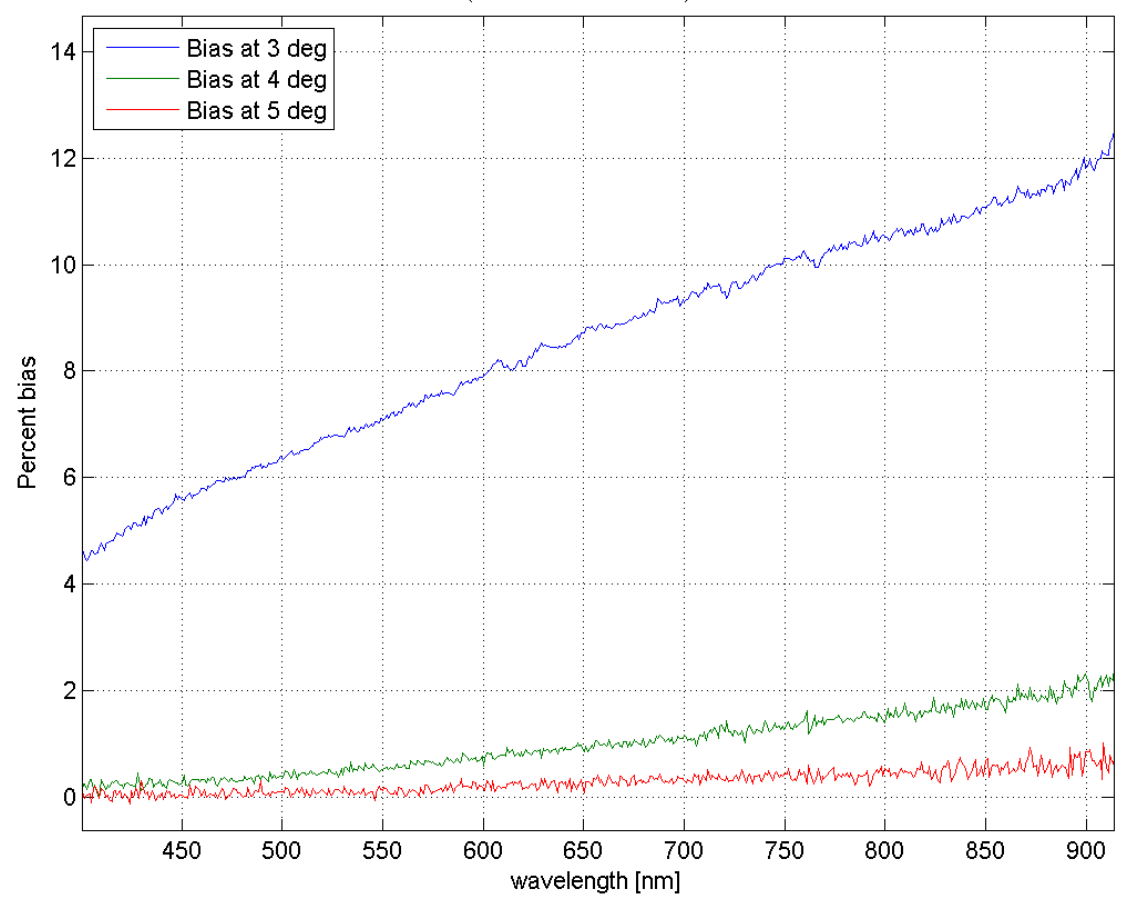

Figure 5. Assessment of 4STAR sky radiance relative to Prede sunphotometer. The shorter collector required for flight operation leads to a reduced ability to exclude stray sunlight and a tendency to positively biased sky radiance measurements close to the sun. The blue, green, and red traces represent the bias as a function of wavelength for sky radiance measured at 3 , 4 , and 5 degrees away from the solar disk. 


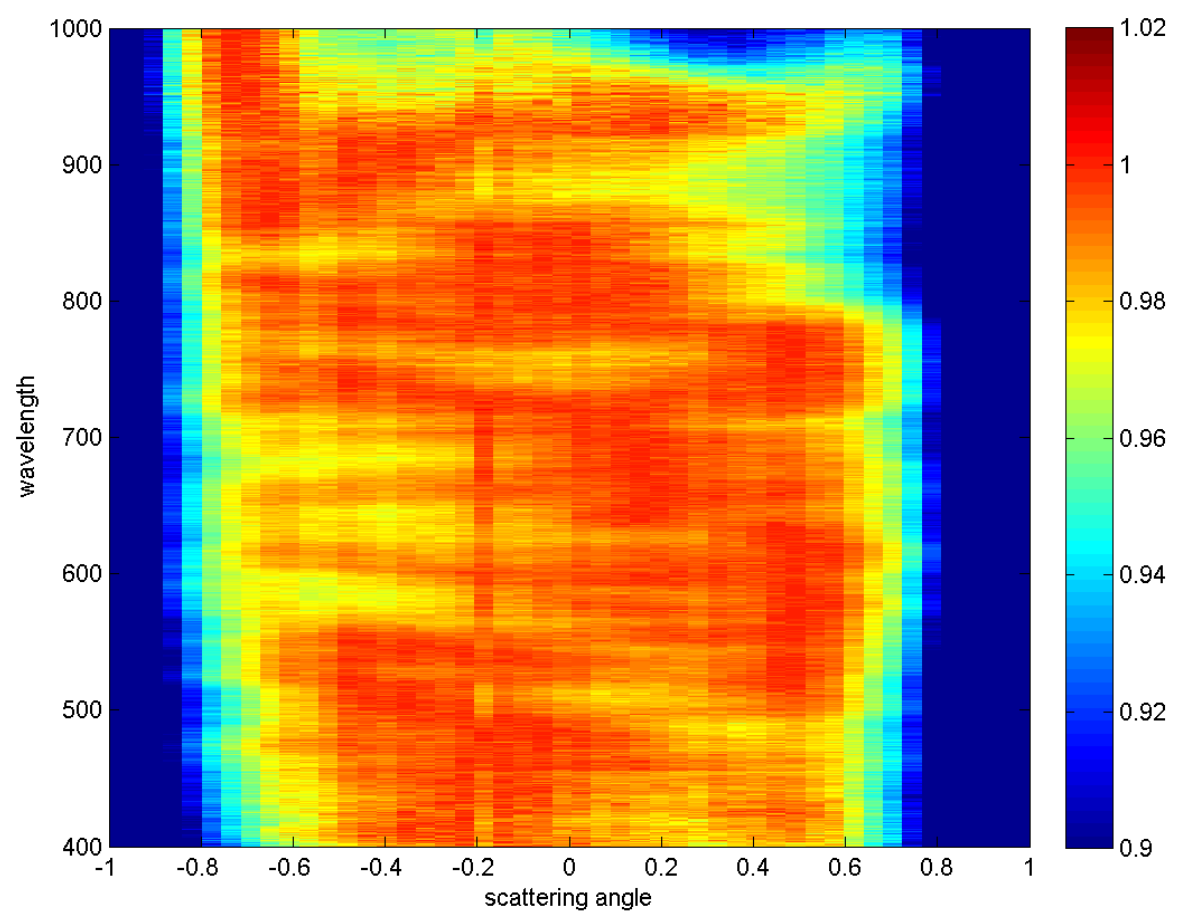

Figure 6. Angular and spectral uniformity of 4STAR direct sun optics. Assessment of the angular uniformity or field-of-view of 4STAR optics required development of new measurement modes and visualization techniques. This false-color plot shows the relative intensity variation as a function of incident angle (on the x-axis) and wavelength (on the y-axis). The color scale has been selected to amplify small deviations in signal response with colors from yellow to dark red spanning about 5\% variability. These results motivated further work to improve the uniformity both angularly and spectrally.

\subsection{Intensive \#4}

The last flight series was conducted the week of August 29, 2011, culminating in successful execution of all desired airborne operational modes including airborne Langley calibration (Figure 7); sun-tracking and sky-scanning within the boundary layer, above the boundary layer, and between elevated aerosol layers; and both passive and active zenith mode for cloud studies. Interestingly, although the presence of volcanic aerosols above the aircraft altitude prevented determination of a "top of atmosphere" calibration, this negative result still convincingly demonstrates the sensitivity of the instrument to even very small aerosol amounts. 

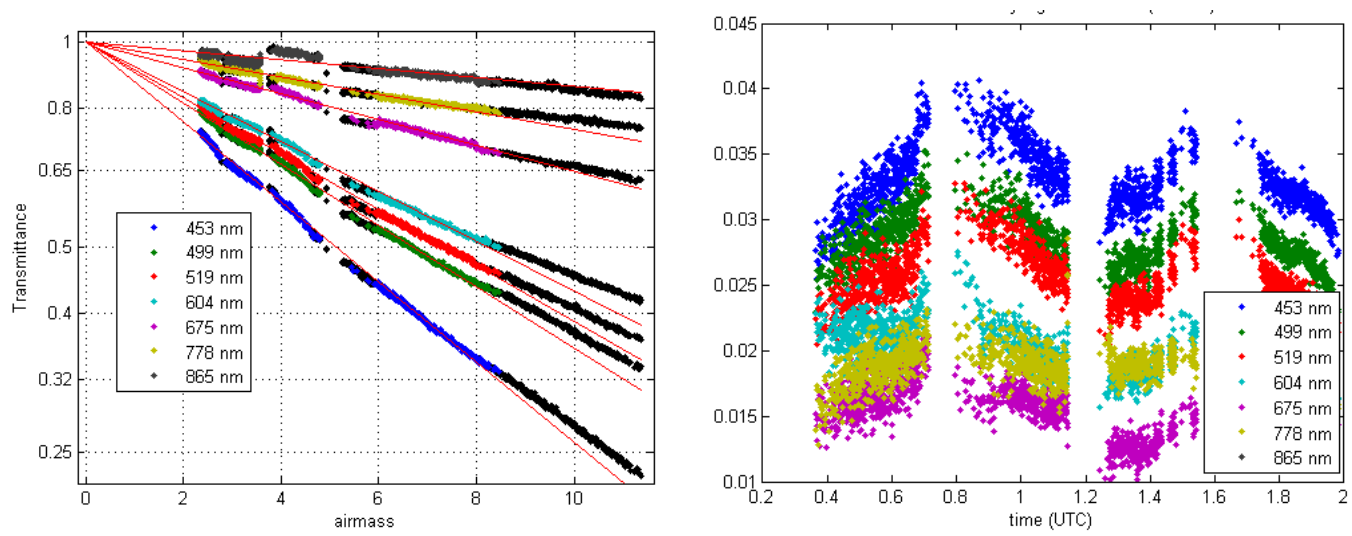

Figure 7. Results of airborne Langley regression. The left-hand plot shows the results from an airborne Langley calibration flight. Atmospheric transmittance is on the vertical axis (on log scale), and the airmass is on the horizontal axis. For uniform atmospheric conditions straight lines are expected. The right-hand plot shows the inferred aerosol optical depth above the instrument varying as a function of time, violating the assumed condition of uniform conditions.

\subsection{Sensitivity Study}

Motivated by the potential for 4STAR sky radiance measurements to experience a positive bias due to incomplete rejection of sunlight, we conducted a sensitivity study to estimate the impact on the retrieved aerosol properties. Calibration errors were represented as a scale-factor offset (either positive or negative) applied to all scattering angles. Bias errors (from incomplete rejection of direct sunlight) were represented as an angularly dependent enhancement. This sensitivity study showed contrasting impacts on the retrieved optical properties (see Figure 8 ) with the optical depth $\tau$ being least affected, the asymmetry parameter g showing most sensitivity to bias (enhancement) errors, and the single-scattering albedo $\omega$ showing more sensitivity to calibration offset.

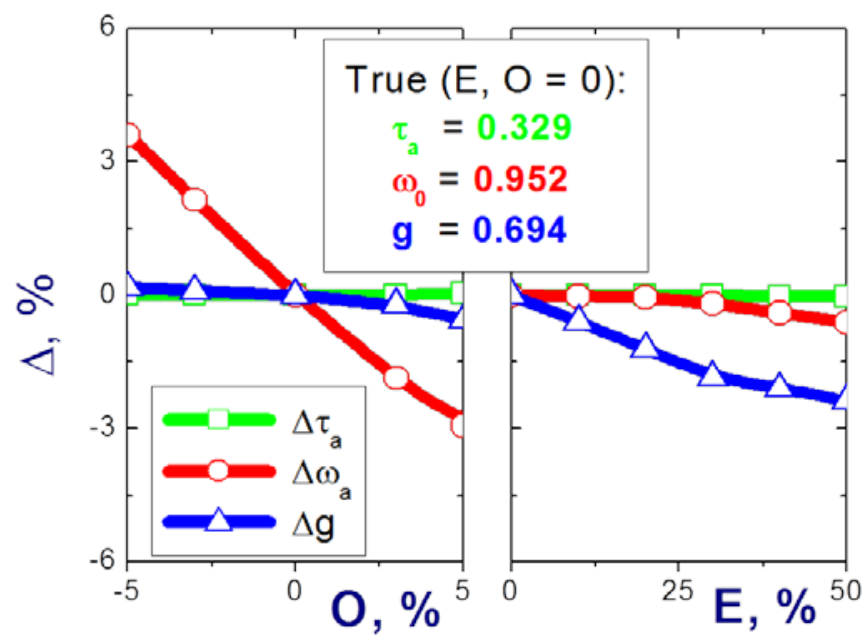

Figure 8. Retrieval sensitivity to sky radiance measurement errors. The left-hand panel shows the effect of calibration error of $\pm 5 \%$ on retrieved aerosol optical properties. The right-hand panel shows the effect of measurement bias. In both panels, green lines indicate errors in optical depth, red lines errors in single-scattering albedo, and blue lines errors in asymmetry parameter. 


\subsection{Technical Readiness Level Advancement}

During the funded period the 4STAR advanced from a TRL of 4 to a final TRL of nearly 8 . These assessments are based on the following NASA definitions of TRL:

TRL 4 Component/subsystem validation in laboratory environment: Standalone prototyping implementation and test. Integration of technology elements. Experiments with full-scale problems or data sets.

TRL 8 Actual system completed and "mission qualified" through test and demonstration in an operational environment (ground or space): End of system development. Fully integrated with operational hardware and software systems. Most user documentation, training documentation, and maintenance documentation completed. All functionality tested in simulated and operational scenarios. Verification and Validation (V\&V) completed.

Subsequently, 4STAR was selected to participate in the ARM TCAP field campaign planned for July 2012 and February 2013 and benefitted from continued maturation efforts in anticipation of this field campaign. 4STAR recently completed an integration test flight for this field campaign where it again demonstrated excellent flight operability, obtained a successful airborne Langley measurement, and demonstrated superior signal levels, solidly establishing a TRL level of 8 or $8+$. It is no exaggeration to suggest that both the selection of 4STAR for TCAP and the current demonstration of science flight capability are directly attributable to the combined support of the 4STAR team through the NASA ROSES proposal and the ARM instrumentation hardening proposal. 


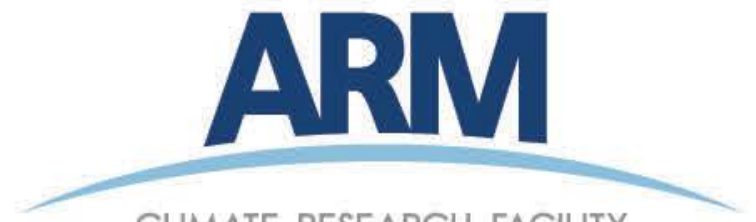

CLIMATE RESEARCH FACILITY

www.arm.gov

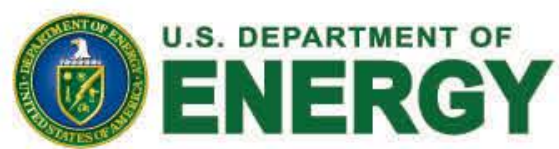

Office of Science 